\title{
Spatiotemporal variation in the long-term fish assemblages of Buck Creek, Delaware County, Indiana
}

\author{
Paul DeRolf ${ }^{1}$, Mark Pyron ${ }^{2}$, and Drew Holloway ${ }^{1}$ \\ ${ }^{1}$ Affiliation not available \\ ${ }^{2}$ Ball State University
}

November 13, 2020

\begin{abstract}
Buck Creek is a spring-fed, cool-water tributary of the West Fork White River, Indiana. The Muncie Bureau of Water Quality sampled fishes and monitored water temperature in Buck Creek annually from 1986-2018. For this study, we utilized long-term fish data from the Bureau of Water Quality to evaluate spatial and temporal changes in the fish assemblages of Buck Creek in Delaware County, Indiana, USA. Non-metric multidimensional scaling (NMDS) was used to describe changes in the fish assemblages over space and time. Linear mixed effects models were used to evaluate the relationship between environmental factors and the fish assemblages. The spatial NMDS results were separated in distinct groups of upstream and downstream assemblages. This was characterized by a shift of headwater specialists shifting to large-river species. The temporal NMDS results were separated into distinct annual assemblages. This was characterized by a drop in pollution-tolerant species and an increase in intolerant species. Our findings indicate that the fish assemblages have improved in Buck Creek over space and time.
\end{abstract}

\section{Hosted file}

DeRolf Manuscript Main Document.pdf available at https://authorea.com/users/375538/ articles/492732-spatiotemporal-variation-in-the-long-term-fish-assemblages-of-buckcreek-delaware-county-indiana 

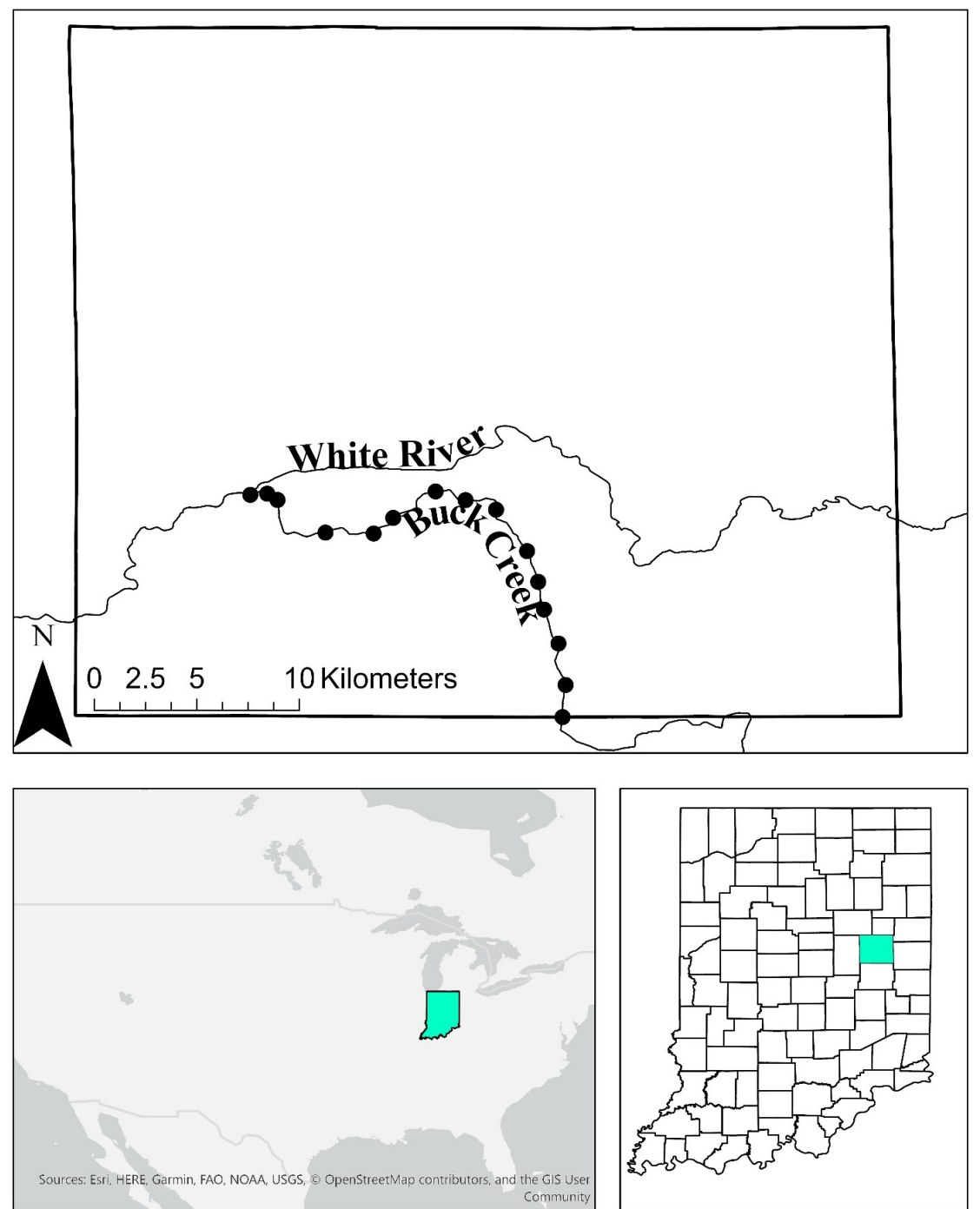

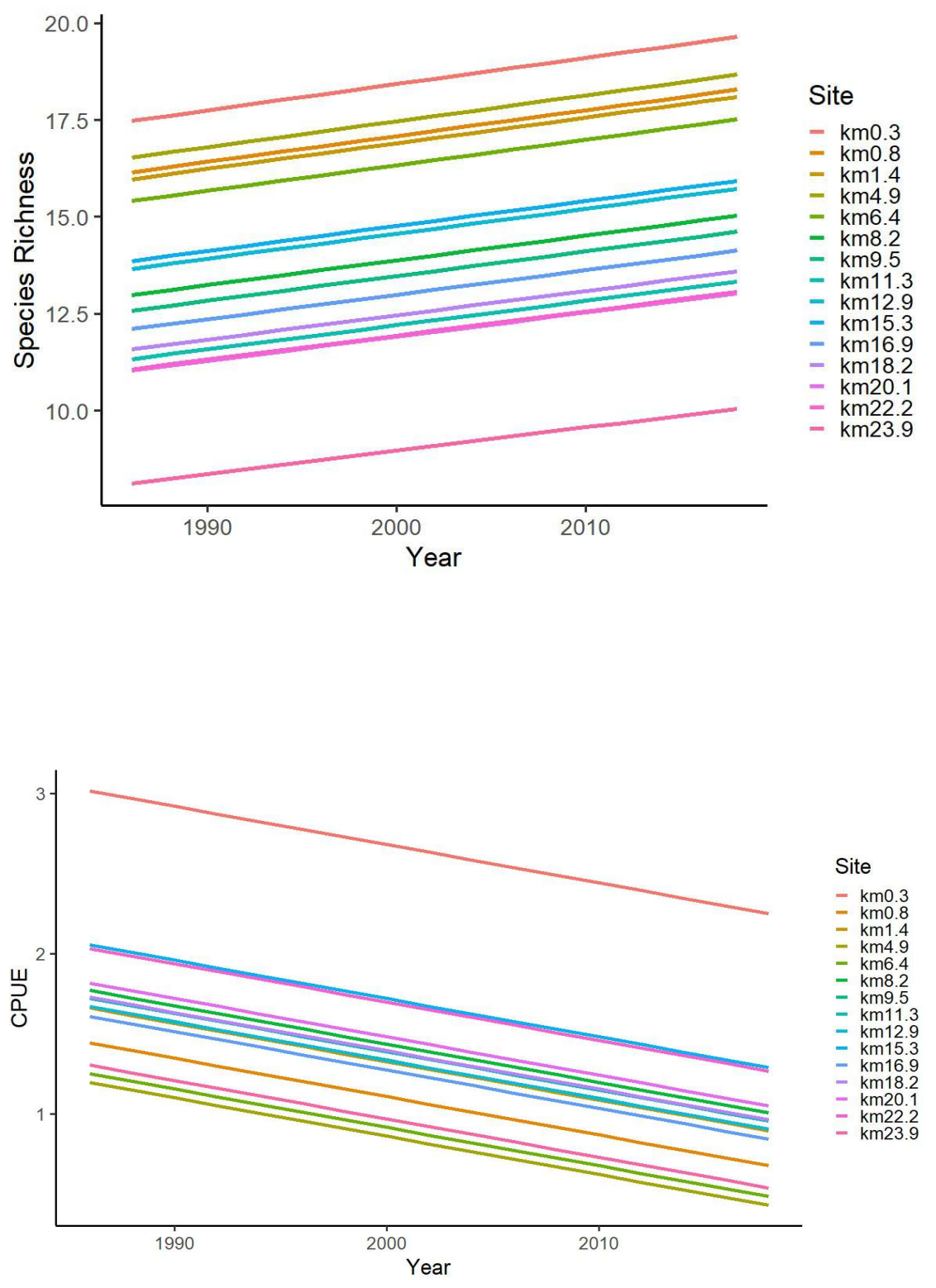

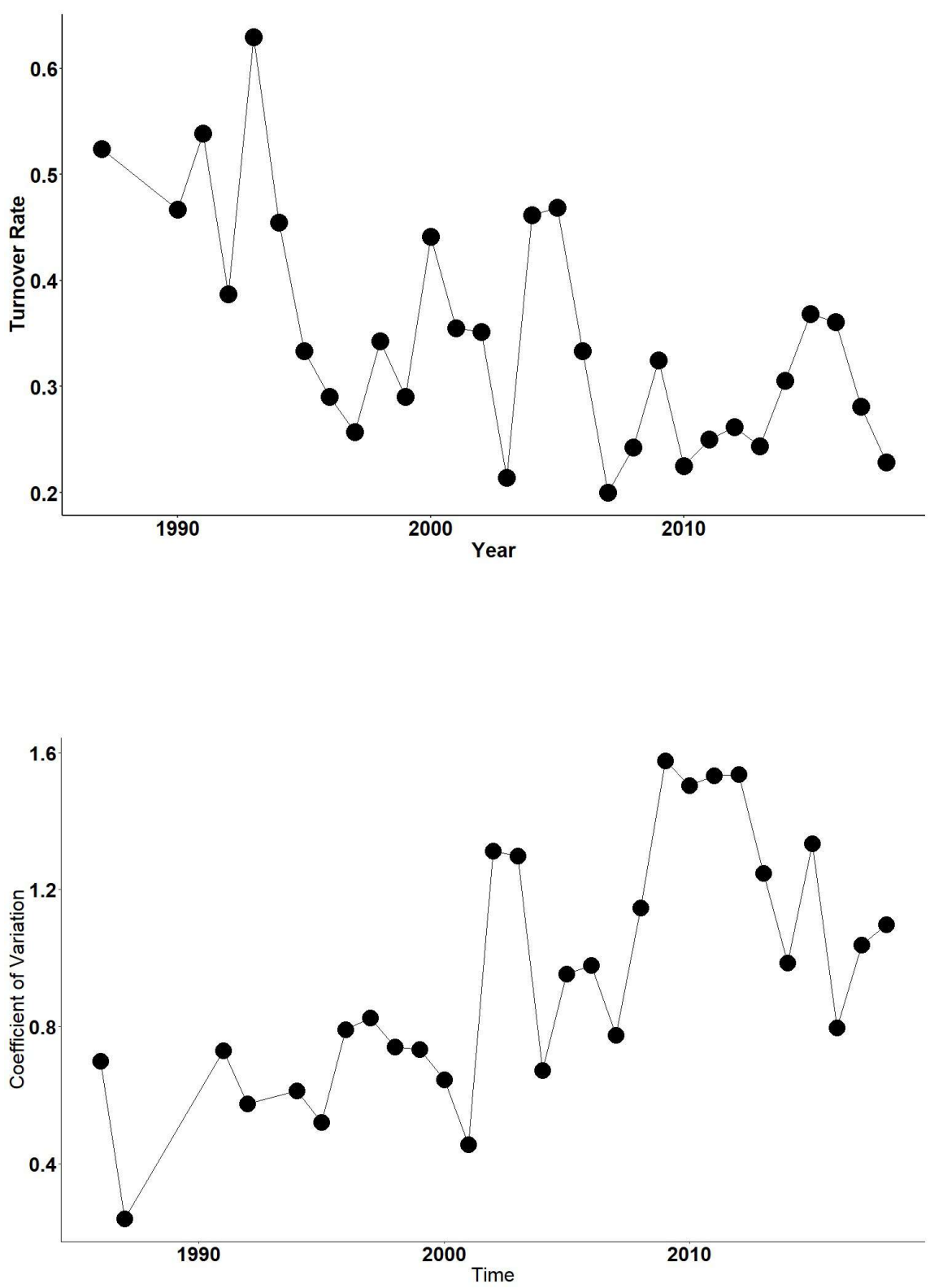

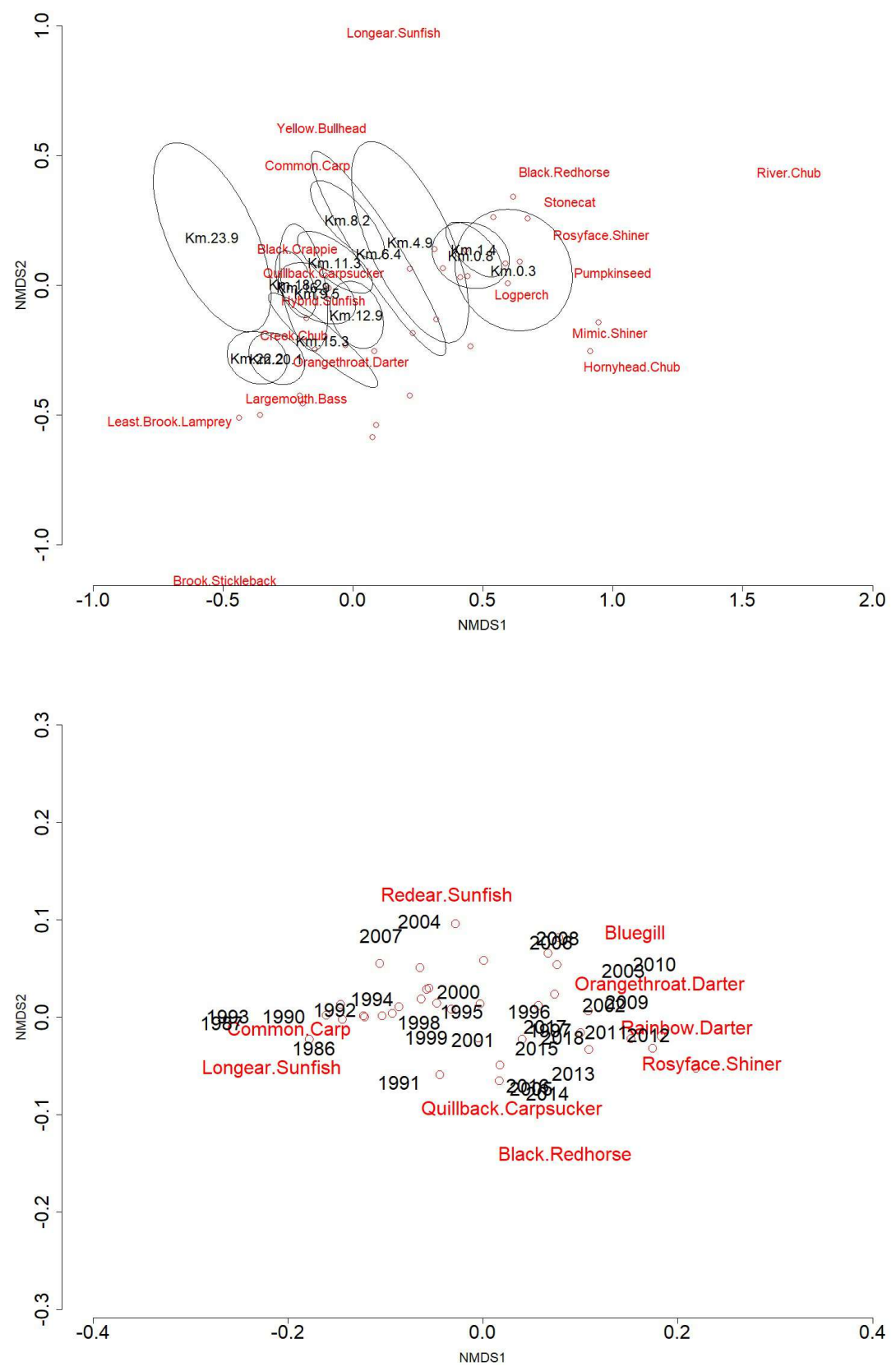

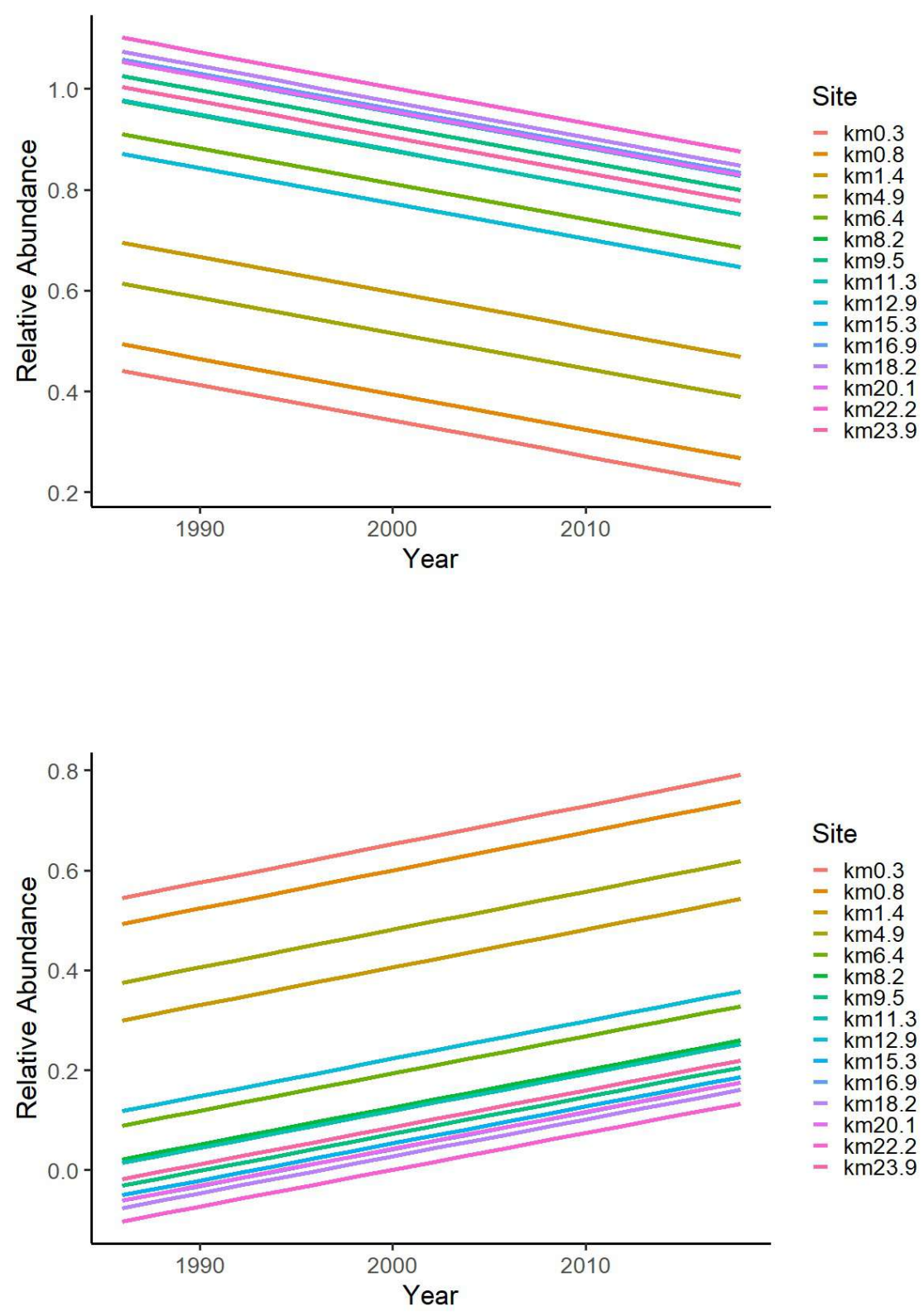


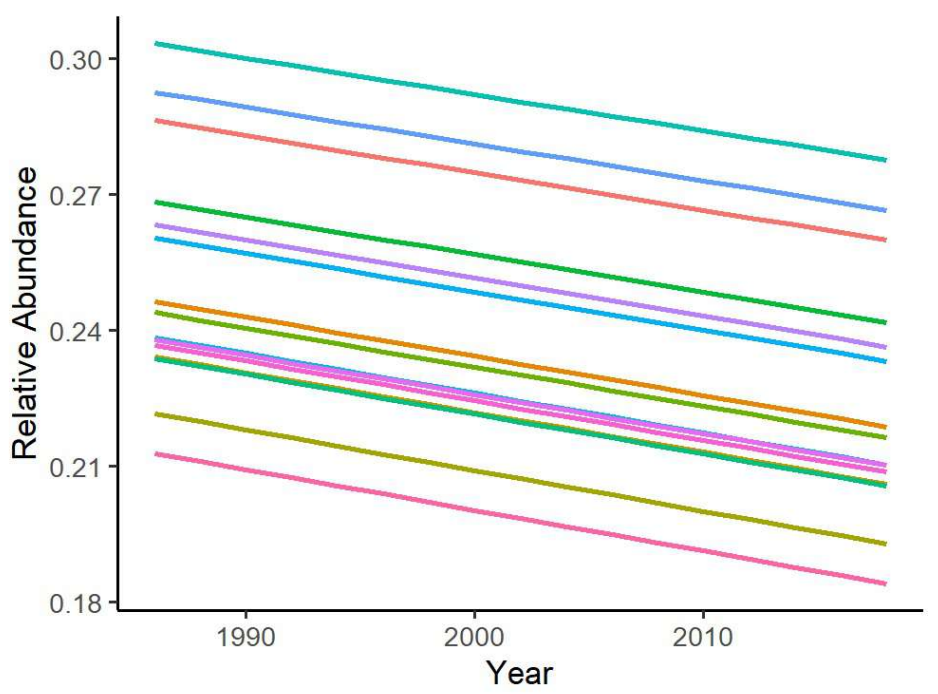

Site

km0.3

- $\mathrm{km0} 8$

- km1.4

- $\mathrm{km} 4.9$

- km6. 4

- km8.2

- km95

- km12.9

- km15.3

- $\mathrm{km} 16.9$

- km18.2

- km20.1

- km22.2

- km23.9

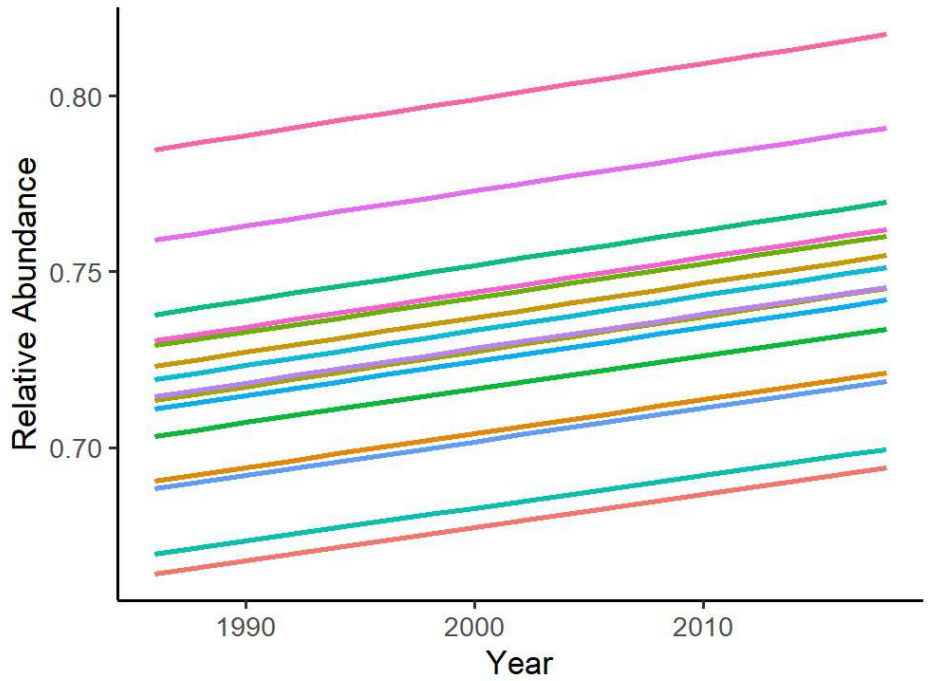

Site

- km0.3

- $\mathrm{km} 0.8$

- km1.4

- km4.9

- km6.4

- km8.2

- $\mathrm{kmg}$

- km11.3

- km12.9

- km15.3

- km16.9

- $\mathrm{km} 18.2$

- km20.1

- km22.2

- km23.9 


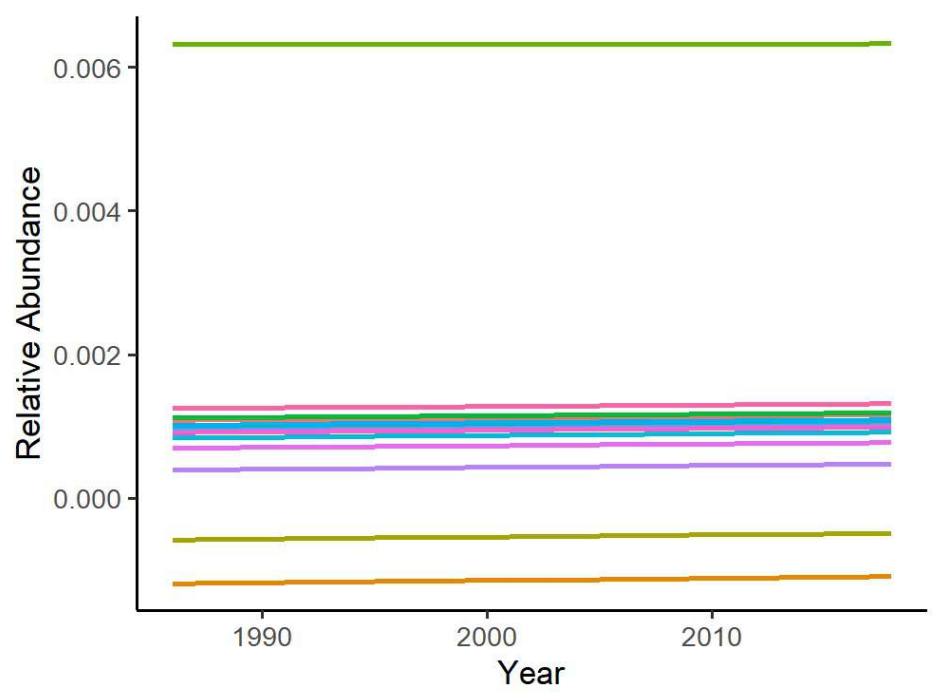

Site

km0.3

- $\mathrm{km0} 8$

- km1.4

- $\mathrm{km} 4.9$

- km6.4

- km8.2

- $\mathrm{km} 95$

- km12.9

- km15.3

- km16.9

- km18.2

- km20.1

- km22

- km23.9

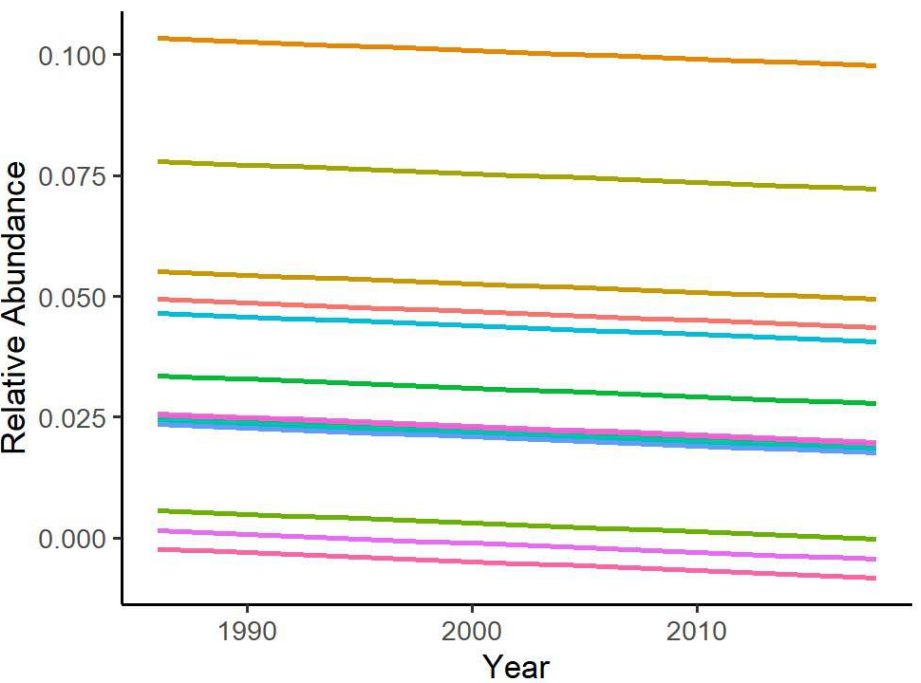

Site

- km0.3

- $\mathrm{km0.8}$

- km1.4

- km4.9

- km6.4

- km8.2

- km9.5

- $\mathrm{km} 11.3$

- km12.9

- km15.3

- km16.9

- $\mathrm{km} 18.2$

- km20.1

- km22.2

- km23.9 


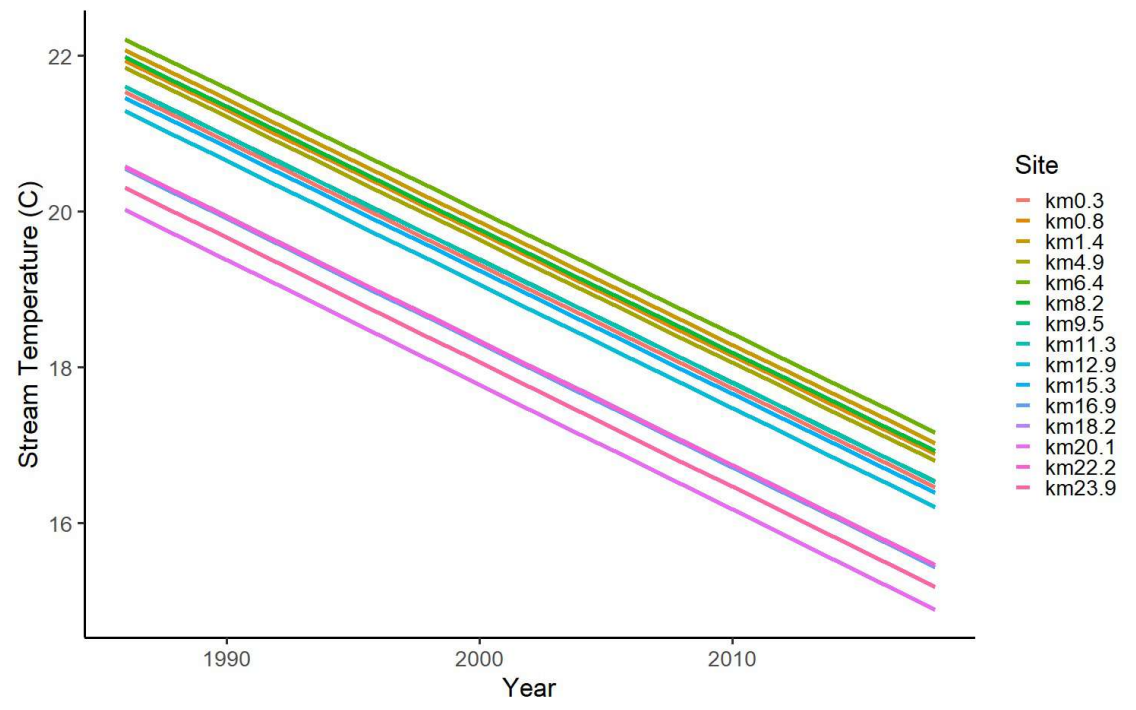

